\title{
TIC, ESTILOS DE APRENDIZAJE Y COMPETENCIA MUSICAL EN LOS ESTUDIOS DE GRADO DE MAESTRO
}

\author{
Ramón Cózar Gutiérrez, $M^{\mathrm{a}}$ del Valle de Moya Martínez, \\ José Antonio Hernández Bravo \& Juan Rafael Hernández Bravo
}

\author{
Universidad de Castilla-La Mancha \\ ramon.cozar@uclm.es, mariavallede.moya@uclm.es, \\ josea.hernandez@uclm.es \& juanrafael.hernandez@uclm.es
}

\begin{abstract}
El desarrollo de las TIC ha traído consigo la difusión global de la música, así como la aparición de nuevas formas de consumo musical. Nuestro objetivo es analizar la competencia musical de los jóvenes (conocimientos, usos y actitudes) tomando como muestra la población universitaria de los Grados de Maestro en las especialidades de Infantil y Primaria ( $n$ =172) de la Facultad de Educación de Albacete, de la Universidad de Castilla-La Mancha. Para ello, se sigue un enfoque cuantitativo con diseño descriptivo mediante la encuesta USMUS. Los resultados revelan que los estudiantes de Primaria muestran conocimientos y usos musicales en relación con las TIC superiores a los de Infantil, al mismo tiempo que le otorgan un papel más destacado para su formación académica por sus implicaciones prácticas.
\end{abstract}

Palabras clave: educación musical, tecnologías de la información y la comunicación, competencias, estrategia de aprendizaje

The development of ICT has brought the global spreading of music and the emergence of new ways to music consumption. Our research aim was to analyze the musical competence of young people (knowledge, skills, and attitudes) taking as sample the university students of Degree $(n=172)$ at the Faculty of Education in Albacete, University of Castilla-La Mancha. We conducted a quantitative approach following a descriptive design by means of USMUS survey. The results reveal that students in Primary Education who used ICT tools show both musical knowledge and uses higher than students in Pre-elementary Education. Besides, students in Primary Education concede to ICT a more prominent role in their academic training due to their practical implications.

Keywords: music education, information and communication technologies, learning strategy, competences

\section{INTRODUCCIÓN}

Las tecnologías de la información y la comunicación (TIC) se erigen como un fenómeno vital para gran parte de la sociedad actual, además de ser las incuestionables señas identitarias de toda una generación. El término "sociedad de la información” es un concepto que apareció hace varias décadas cuando diversos autores profetizaban este nuevo tipo de sociedad (por ejemplo, Machlup, 1962; Touraine, 1969; Bell, 1973; Bangemann, 1994; Castells, 1998) y la transformación de sus actividades humanas (economía, cultura, política, ocio, educación) por influencia de las TIC (Giráldez, 2005). Su constante e inevitable presencia en la vida cotidiana es causa y efecto a la vez de profundos cambios en las relaciones humanas, al originar nuevas situaciones laborales, de estilos de vida, de pensamiento y transmisión del conocimiento e incluso de interdependencia. Hablar de las TIC nos remite a un macro procedimiento en el que se integran medios de comunicación y procesamientos de la información, mediante un proceso de digitalización que permite la unificación de códigos y formatos (Majó \& Marqués, 2002). Internet y sus contenidos digitales se han convertido en la mayor biblioteca que ha conocido el mundo, pero obliga a una necesaria y permanente actualización continua en lo que se refiere a técnicas documentales específicas para no perderse en el ritmo frenético de los cambios y avances que no cesan de producirse (Ramos, 2011). Por todo ello, podemos afirmar que el ciudadano del siglo XXI vive en un "entorno personal virtualizado" que fluctúa en un constante proceso de cambio, de transformación, perfeccionamiento, proliferación e innovación característico del mundo de las TIC, impactante y revolucionario fenómeno técnico y social, que afecta a todas las actividades humanas, laborales, formativas, académicas, de ocio y consumo.

Para diversos autores (Cuban, 2001; Kerr, 2004; McMillan, Honey \& Mandinach, 2005), estas herramientas tecnológicas mejoran las prácticas profesionales ya que poseen un carácter interactivo que otorga a los usuarios de las nuevas tecnologías un papel más activo en la profundización de la información que con los medios de comunicación tradicionales. Además, las TIC son instantáneas, 
posibilitan la interconexión entre tecnologías y personas y penetran de una forma significativa en todos los sectores sociales, fomentando el concepto de globalización (Hernández, 2013). Poseen un estelar protagonismo incluso en aquellos campos considerados más tradicionales y algo reacios a los cambios, como es el caso de la educación.

En el contexto educativo, las herramientas digitales han revolucionado las metodologías pedagógicas en un corto espacio de tiempo. Así, se han superado las barreras físicas del aula convencional gracias al desarrollo de entornos de aprendizaje virtuales, que ha ido en paralelo a un cambio de mentalidad hacia los beneficios de las TIC y, al mismo, ha generado una necesidad de formación al objeto de adquirir conocimientos y habilidades para su correcta alfabetización digital. Para Ricoy, Feliz y Sevillano (2010), la incorporación de las nuevas tecnologías en educación ha originado el desarrollo de nuevas competencias por parte de los estudiantes para relacionarse con objetos de conocimiento en estos entornos digitales, suponiendo parte del fenómeno de transformación de las prácticas sociales, y entre ellas las educativas. No obstante, la novedad pedagógica de estas herramientas ha puesto de manifiesto carencias en su uso y malas prácticas con lo que se deja de aprovechar todas las bondades que las TIC ofrecen. En este sentido, para De Moya y Cózar (2013, p. 10):

Está más que justificada la inclusión de la Competencia en los currículos de los diferentes niveles educativos, ya que los alumnos, reflejo de su tiempo vital histórico, son unos fieles usuarios tecnológicos, pero no son competentes en el uso y tratamiento de las TIC. De esta manera, se impone la necesidad de capacitarlos para que lleguen a ser competentes digitales en todas las facetas y actividades de su vida adulta. $Y$ en este sentido, el centro educativo y los docentes desempeñan un papel imprescindible.

En un mundo cada vez más complejo dominado por la tecnificación, se hace necesario el empleo de competencias, tanto para docentes como para el alumnado. Enseñar y aprender a trabajar por competencias requiere una necesaria renovación en los procesos pedagógicos, en los que el tratamiento de la información y la competencia digital puede ayudar a afrontar con éxito las situaciones de aprendizaje actuales, desarrollando para ello un espíritu crítico ante los mensajes que se reciben. En este marco competencial, el profesorado se erige como una figura clave en el proceso integrador de las TIC y en un actor imprescindible para abordar el proceso de enseñanza-aprendizaje aplicando aquellos recursos tecnológicos que más se ajusten a sus objetivos didácticos. Ahora bien, este hecho conlleva no sólo que el docente domine las herramientas TIC, sino que sepa seleccionar los recursos que precisa, de qué forma y cuándo ha de utilizarlos en su práctica docente diaria (Suárez, Almerich, Gargallo \& Aliaga, 2013).

Una válida definición de las muchas existentes sobre la Competencia Digital es la que nos ofrece Mir (2010) al entender que es "la combinación de conocimientos, habilidades y capacidades, en conjunción con valores y actitudes, para alcanzar objetivos con eficacia y eficiencia en contextos y con herramientas digitales”. Así, la legislación imperante entiende que el Tratamiento de la Información y Competencia Digital "consiste en disponer de habilidades para buscar, obtener, procesar y comunicar información y para transformarla en conocimiento”. Se pretende desarrollar habilidades que permitan el acceso a una información útil hasta su adecuada transmisión en distintos soportes (oral, impreso, audiovisual, digital o multimedia), empleando las TIC como medio esencial para informarse, aprender, disfrutar y comunicarse.

Ser competente en la utilización de las TIC supone saber utilizarlas en su doble función: por un lado, como trasmisoras y generadoras de información y conocimiento; por otro, como apoyo formativo a los procesos lingüísticos, matemáticos, sociales o artísticos. Y aún nos atrevemos a añadir otra línea de utilización: la de la comunicación social y el ocio y entretenimiento (en grupo o individualmente). En síntesis, se pretende desarrollar una persona que sea autónoma, eficaz, responsable crítica y reflexiva al seleccionar, tratar y utilizar las información y sus fuentes, así como las distintas herramientas tecnológicas con todas las posibilidades que ofrecen para el uso de relaciones humanas y sociales.

Cada época está marcada por unos parámetros culturales y sociales que condicionan la producción 
y las actividades humanas de ese momento. En este sentido, la obra de arte es fiel reflejo del momento histórico que la ha visto nacer. Según Eco (2001, p. 35), en las artes “están presentes los modos de pensar, vivir y sentir de toda una época, la interpretación de la realidad, la actitud frente a la vida, los ideales y las tradiciones, las esperanzas y la luchas de una etapa histórica”. El arte es una expresión de la actividad humana caracterizada por la interacción de diferentes lenguajes. Para Hernández (2013, p. 139):

El arte constituye un medio ideal para la comunicación entre los seres humanos, facilitando al mismo tiempo el desarrollo de su inteligencia y su capacidad expresiva. Los medios tradicionales de expresión artística han sido la pintura, la escultura, el dibujo, la música, la literatura. Posteriormente, se añadió la fotografía y el cine, y, en los últimos tiempos, las TIC.

En el devenir de los tiempos, la música ha sido considerada como un lenguaje universal y un excelente vehículo de actividad social, ya que permite la comunicación, la participación y el intercambio de ideas. Como manifestación humana, la música ha tenido una gran importancia en cualquier cultura y civilización, trascendiendo el tiempo y el espacio y constituyendo un fenómeno transcultural al igual que el lenguaje o las emociones (Bermell \& Alonso, 2012). Así, cada generación se ha identificado con unas determinadas preferencias culturales que hace suyas, siendo la música un excelente medio de expresión de las aspiraciones y señas de identidad de la misma a lo largo del tiempo. Desde mediados del siglo XX, la juventud ha visto en la música una forma de identificarse con su propia “cultura urbana”, además de diferenciarse de la generación anterior. La industria del ocio, partiendo de esta realidad, ha puesto en marcha una serie de mecanismos para fomentar el consumo musical, estableciendo formatos y usos de diversión musicales como requisitos necesarios para la socialización de la juventud. Según De Aguilera y Sedeño (2008), la música ha buscado nuevas formas de comunicación, por lo que se hace necesario conocer su dimensión expresiva y, al mismo tiempo, comprender cómo estas vías de comunicación han transformado para siempre la manera de producción y usos musicales.

Las TIC ofrecen a los jóvenes una oportunidad para responder con la calidad y eficiencia necesarias a una demanda, cada vez más masiva y diversificada, que se reclama desde todas las áreas de conocimiento, y, entre ellas, la música (Hernández, 2013). En cualquier caso, parece una evidencia demostrada que las TIC se alzan como el medio, el canal de expresión, más desarrollado y extendido y, por supuesto, el más importante de los años que llevamos de siglo.

En la actualidad, se observa cómo la música, su difusión y su consumo, aparece fuertemente asociada al desarrollo de las TIC, verdadero fenómeno revolucionario, técnico y social que impregna todas las actividades juveniles, tanto académicas como de ocio y consumo, incrementándose la predisposición de los estudiantes hacia la música y hacia los procesos creativos musicales (Gértrudix \& Gértrudix, 2014). En este sentido, Aranguren (2011) resalta el gran impacto que ha supuesto la implantación de las TIC en la vida cotidiana de los jóvenes, y en concreto, en el ámbito musical, poniendo de relieve la enorme disparidad de músicas a las que tienen acceso a través de Internet, una situación que condiciona sus hábitos y comportamientos hacia el hecho musical. Gracias a las tecnologías modernas, que se encuentran en constante proceso de cambio y superación (Storr, 2008), es posible escuchar cualquier tipo de música en el momento que se desee, sobre todo, la música comercial o música de consumo, que suele ser la más extendida, escuchada, usada y transmitida por medio de las TIC.

Campos (2008) considera que la música, apoyada en la tecnología, transmite más de lo que dice explícitamente como discurso artístico, no está al margen de los cambios sociales y siempre se transfiere en interacción con distintas realidades. Por lo tanto, la clave para evitar el determinismo tecnológico se encuentra en reflexionar acerca de la interacción entre tecnología y contexto, en comprender sus propiedades y en entender cómo puede ser utilizada en la práctica para mejorar los procesos didácticos (Ruthmann, Tobias, Randles \& Thibeault, 2015). Además, el empleo del hecho musical a través de las herramientas tecnológicas supone la ruptura de fronteras espaciales, temporales o estilísticas, entre otras, ofreciendo al usuario mayores posibilidades de formación 
(Blardony, 2006). En esta línea, Torres (2010) destaca la necesaria formación de los docentes de música para conocer las tecnologías que continuamente van surgiendo en educación, ser conscientes de su potencial y, sobre todo, para saber integrarlas en el proceso de enseñanza-aprendizaje.

El análisis del consumo y la competencia musical (conocimientos, usos y actitudes) que se hace de este consumo a través de las TIC ponen en evidencia una escasez de estudios al respecto para determinar la forma en que convergen estos campos. Las prácticas personalizadas de consumo, el perfeccionamiento de los dispositivos tecnológicos para el consumo musical, el intercambio cada vez más creciente de contenidos musicales dentro de la red en una sociedad globalizada, así como una tendencia imparable en el aumento de estos contenidos, revelan nuevas prácticas musicales y un tema de plena vigencia y de interés científico para nuestra investigación. Los medios digitales no han transformado la música preexistente, pero han propiciado la aparición de una nueva cultura de consumo musical alrededor de estos dispositivos en la que las nuevas plataformas digitales ofertan al público receptor un amplio abanico de posibilidades referidas al acceso, copia y tratamiento del sonido musical, siendo formas de consumo musical individualizadas. Ahora bien, más allá de una finalidad lúdica o un medio de difusión, las TIC aplicadas a la enseñanza musical han de entenderse como unas herramientas con un notable poder didáctico, tanto en la creación como en la producción, que sirven para generar y compartir contenidos musicales en una mundo globalizado e interconectado (Burnard, 2007).

A diferencia de generaciones anteriores, la juventud actual ha crecido rodeada del hecho tecnológico, factor que ha favorecido enormemente su comprensión de las TIC, así como el desarrollo de habilidades para su manejo, hecho que contrasta fuertemente si se compara con sus antepasados. En el ámbito universitario, los estudiantes pertenecen a esta joven generación tecnológica y emplean las TIC de una forma habitual en sus vidas, tanto académica como social. En este contexto, los estudiantes universitarios están familiarizados con el uso de las TIC y las nuevas formas de consumo propiciadas por ellas, entre las que se encuentra el consumo musical. Para profundizar en esta realidad, se han realizado diversos estudios (Anderson \& Tracey, 2002; Buckingham, 2002; Livingstone, 2009; Tapscott, 2009) que han analizado el impacto de las TIC en la vida diaria de los estudiantes y, de manera más concreta, han determinado la influencia decisiva de las herramientas tecnológicas en el consumo musical actual (Aranguren, 2011; Herrera, Cremades \& Lorenzo, 2010; Solomando, Almerich \& Fossati, 2012). Además, otras investigaciones (Barry, 2004; Bauer, McAllister \& Reese, 2003; Beckstead, 2001; Enders, 2000; Ho, 2004; Savage, 2005), que han estudiado las percepciones y actitudes de los universitarios que emplean las TIC en el conocimiento y uso de la música, han puesto de manifiesto que este alumnado otorga un bajo potencial didáctico de estas herramientas, quedando todavía lejana la integración efectiva de las TIC en los procesos educativos musicales.

Los estudios emprendidos hasta la fecha con frecuencia se han centrado en la descripción del fenómeno sociológico música-TIC, si bien es relevante profundizar en la competencia musical, entendida en término de conocimientos, usos y actitudes musicales, tratando de determinar si el estilo de aprendizaje del alumnado universitario condiciona el consumo que hace de la música mediante las nuevas tecnologías. Con ello, se pretende buscar correlaciones entre las formas particulares de aprender que tiene cada persona (Alonso \& Gallego, 2003) y sus experiencias musicales a través de las TIC. En esta línea, una investigación realizada por De Moya, Hernández, Hernández y Cózar (2011) analizó las relaciones entre conocimientos y usos de las TIC vinculados con el estilo de aprendizaje predominante en el alumnado universitario. Este trabajo, que presentaba la validación del instrumento REATIC, sirvió de base para la investigación recogida en el presente artículo, en el que se amplía el ámbito de estudio a la práctica musical relacionada con las TIC y el estilo de aprendizaje. USMUS y REATIC son cuestionarios creados por los autores del presente artículo para realizar estudios con un enfoque cuantitativo y diseño no-experimental, sobre una escala tipo Likert, basados en las TIC. USMUS analiza el consumo musical a través de las TIC y REATIC se centra en establecer el grado de conocimiento y uso de las TIC. Ambos se relacionan con los estilos de aprendizaje del 
alumno universitario.

El punto de partida de nuestra investigación fue el planteamiento de diferentes cuestiones: ¿qué lugar ocupa la música en la vida cotidiana de los jóvenes?, ¿cómo la usan, consumen y comparten dentro de su grupo social a través de las redes virtuales?, ¿puede existir una vinculación entre la predisposición, actitud y valoración de los usos musicales y el empleo de las TIC entre la juventud?, ¿es posible que exista una relación (que influya o condicione) el estilo de aprendizaje predominante en cada individuo con la forma de llevar a cabo diversas actuaciones musicales tecnológicamente?, Y, si así fuera, ¿de qué modo se haría?

Conocer el tipo de música que escuchan los jóvenes y el medio tecnológico que emplean para ello, ofrecerá a los docentes una valiosa información para determinar los conocimientos y actitudes de los estudiantes hacia la música. Si a este hecho se le añade que dicho consumo musical por medio de las TIC puede estar vinculado a un estilo de aprendizaje predominante (activo, reflexivo, teórico o pragmático) el docente podrá establecer estrategias de actuación para mejorar dicho consumo. De este modo, el profesorado tendrá la suficiente información para establecer unas determinadas actividades musicales que posean mayor calidad artística; utilizar diferentes y mejorados dispositivos tecnológicos, fácilmente accesibles para la población juvenil; demostrar cómo se puede hacer un mejor uso de la música; potenciar diferentes procesos y estrategias de aprendizaje musical, combinando los ejes recepción-descubrimiento y significativo-memorístico (Rusinek, 2004), así como fomentar un profundo conocimiento personal y un desarrollo creativo en diversos momentos de su vida cotidiana.

\section{OBJETIVOS}

Considerando el estado de la cuestión descrito en líneas anteriores, el presente estudio recoge los siguientes objetivos de investigación:

- Analizar la competencia musical (conocimientos, usos y actitudes) en el alumnado universitario de la Facultad de Educación de Albacete vinculada con las TIC y su estilo de aprendizaje en función de la especialidad del Grado de Maestro (Educación Infantil o Primaria).

- Determinar si los conocimientos, usos y actitudes musicales relacionados con las TIC y el estilo de aprendizaje difieren en función del género de los estudiantes de dicha Facultad.

\section{MÉTODO}

En este estudio se siguió un enfoque de tipo cuantitativo para dar respuesta a los objetivos de investigación planteados. Este enfoque se caracteriza por ser explicativo, predictivo y de control, con la intención de revelar por qué suceden los fenómenos a través de las evidencias observadas, la recopilación de datos y el análisis de los mismos (Cardona, 2002). Se optó por un diseño no experimental descriptivo mediante encuesta, un tipo de diseño muy utilizado en metodología cuantitativa para conocer la realidad estudiada y preparar futuras investigaciones. Tras un estudio exploratorio previo en la Facultad de Educación de Albacete, Universidad de Castilla-La Mancha, se apreció que era generalizado el uso y consumo musical de los jóvenes universitarios empleando las herramientas tecnológicas, por lo que se consideró a estos estudiantes como candidatos para participar en una investigación más profunda. La muestra ascendió a 172 participantes (149 mujeres, 86\%; 23 varones, 14\%) del Grado de Maestro de Infantil (83 estudiantes, 48\%) y Primaria (89 estudiantes, 52\%) de la mencionada Facultad. El acceso a los participantes fue por disponibilidad y accesibilidad muestral, realizándose la investigación en el segundo trimestre del curso 2013-2014.

Para la medición de las variables estudiadas (conocimientos, usos, actitudes musicales en relación con las TIC y con el estilo de aprendizaje), se administró el instrumento USMUS (De Moya, Hernández, Hernández \& Cózar, 2013), con cuatro opciones de respuesta tipo Likert (de Nada a 
Mucho). El instrumento original fue sometido a un análisis factorial exploratorio con la intención de reducir el número de ítems y reagruparlos en dimensiones o factores, tratando de perder la mínima información posible. De este modo, se delimitaron cuatro factores: conocimientos, usos, actitudes musicales, así como usos y actitudes musicales según el estilo de aprendizaje (véase Tabla 1), que se correspondían con los cuatro subapartados del instrumento. Tras efectuar el análisis factorial de componentes principales del cuestionario, los ítems se redujeron de 44 a 20. Como prueba de consistencia interna del instrumento, se calculó el Alpha de Cronbach, obteniéndose un coeficiente de 0.88 , lo que confirmó la consistencia de los ítems. La evidencia acerca de la validez de constructo se obtuvo a partir del análisis factorial empleando el método de análisis de los componentes principales. Los índices obtenidos en las pruebas de adecuación muestral $(\mathrm{KMO}=0,78)$ y esfericidad de Barlett, $\chi^{2}(3182)=566, p=0,000$, resultaron adecuados para proceder con el análisis. Para el análisis de los datos se calcularon estadísticos descriptivos (media y desviación típica) y correlacionales (ANOVA), empleando para ello el paquete informático SPSS versión 19 para Windows.

\begin{tabular}{|c|c|c|c|c|}
\hline Items & Factor & Factor II & Factor III & Factor IV \\
\hline \multicolumn{5}{|l|}{ Conocimientos musicales } \\
\hline Blog, wiki, foro para comentar música & 0,74 & & & \\
\hline Medios para grabar compartir música & 0,72 & & & \\
\hline Portales de vídeo musicales online & 0,68 & & & \\
\hline Dispositivos multimedia interactivos & 0,61 & & & \\
\hline \multicolumn{5}{|l|}{ Alpha $=0,71$} \\
\hline \multicolumn{5}{|l|}{ Autovalores $=3,6$} \\
\hline \multicolumn{5}{|l|}{ Varianza explicada $=20,12 \%$} \\
\hline \multicolumn{5}{|l|}{ Usos musicales } \\
\hline Reproductores de sonido & & 0,78 & & \\
\hline Portales de vídeo musicales online & & 0,72 & & \\
\hline Enciclopedias virtuales musicales & & 0,60 & & \\
\hline Dispositivos multimedia interactivos & & 0,58 & & \\
\hline \multicolumn{5}{|l|}{ Alpha $=0,75$} \\
\hline \multicolumn{5}{|l|}{ Autovalores $=3,2$} \\
\hline \multicolumn{5}{|l|}{ Varianza explicada $=18,65 \%$} \\
\hline \multicolumn{5}{|l|}{ Actitudes musicales } \\
\hline Importante conocer música desde niño & & & 0,81 & \\
\hline Medio para fomentar relaciones & & & 0,74 & \\
\hline Fácil acceso gracias a las TIC & & & 0,72 & \\
\hline Debe conservarse el folclore & & & 0,64 & \\
\hline \multicolumn{5}{|l|}{ Alpha $=0,68$} \\
\hline \multicolumn{5}{|l|}{ Autovalores $=3,5$} \\
\hline \multicolumn{5}{|l|}{ Varianza explicada $=21,13 \%$} \\
\hline \multicolumn{5}{|l|}{ Usos y actitudes según estilo de aprendizaje } \\
\hline Me gusta buscar música en la red & & & & 0,68 \\
\hline Descubro músicas nuevas a amigos & & & & 0,64 \\
\hline Me gusta organizar tertulias musicales & & & & 0,59 \\
\hline Pienso que los debates musicales unen & & & & 0,57 \\
\hline Prefiero investigaciones musicales en solitario & & & & 0,57 \\
\hline Actúo con intuición en comentarios musicales & & & & 0,55 \\
\hline Me interesa opiniones musicales de otros & & & & 0,54 \\
\hline Obtengo conclusiones en trabajos musicales & & & & 0,52 \\
\hline \multicolumn{5}{|l|}{ Alpha $=0,70$} \\
\hline \multicolumn{5}{|l|}{ Autovalores $=3,8$} \\
\hline Varianza explicada $=16,22 \%$ & & & & \\
\hline
\end{tabular}

Tabla 1: Estructura factorial del cuestionario USMUS 


\section{RESULTADOS}

A continuación ofrecemos los resultados obtenidos agrupados por objetivos de investigación.

1) Analizar la competencia musical (conocimientos, usos y actitudes) en el alumnado universitario de la Facultad de Educación de Albacete vinculada con las TIC y su estilo de aprendizaje en función de la especialidad del Grado de Maestro (Educación Infantil o Primaria).

Se observaron diferencias estadísticamente significativas al 5\% o superior en 8 ítems, siendo muy significativas $(p=0,000)$ en dos de ellos: "Conozco dispositivos multimedia interactivos" $(M=2.31$; $D T=0,89)$ y "Uso dispositivos multimedia interactivos" $(M=2.11 ; D T=0,89)$, ambos a favor del Grado de Primaria. En los otros siete ítems se apreciaron las siguientes diferencias estadísticamente significativas. A favor del Grado de Infantil, en el subapartado actitudes se hallaron los ítems: "Es importante conocer la música desde niño" $(M=3.51 ; D T=0,57)(p=0,014)$; "La música es un medio para fomentar relaciones" $(M=2.81 ; D T=0,80)(p=0,040)$; $\mathrm{y}$ "Debe conservarse el folclore" $(M=$ 3.05; $D T=0,71)(p=0,030)$. En el Grado de Primaria aparecieron diferencias significativas en los ítems "Uso portales de vídeo musicales online" $(M=3.33 ; D T=0,75)(p=0,031)$; "Pienso que los debates musicales unen” ( $M=2.65 ; D T=0,79)(p=0,009)$; y “Obtengo conclusiones en trabajos musicales” $(M=2,39 ; D T=0,83)(p=0,025)$. La Tabla 2 recoge la comparación de medias por especialidad de Grado.

\begin{tabular}{|c|c|c|c|c|c|c|}
\hline & & $\mathbf{M}$ & DT & $\mathbf{F}$ & gl & $\mathbf{p}$ \\
\hline \multicolumn{7}{|l|}{ Conocimientos musicales } \\
\hline \multirow{2}{*}{ Blog, wiki, foro para comentar música } & $\mathrm{I}$ & 1,96 & 0,91 & \multirow{2}{*}{0,67} & \multirow{2}{*}{171} & \multirow{2}{*}{0,414} \\
\hline & $\mathrm{P}$ & 2,08 & 0,92 & & & \\
\hline \multirow{2}{*}{ Medios para grabar compartir música } & $\mathrm{I}$ & 2,53 & 0,73 & \multirow{2}{*}{0,01} & \multirow{2}{*}{171} & \multirow{2}{*}{0,914} \\
\hline & $\mathrm{P}$ & 2,52 & 0,85 & & & \\
\hline \multirow{2}{*}{ Portales de vídeo musicales online } & $\mathrm{I}$ & 3,20 & 0,67 & \multirow{2}{*}{0,84} & \multirow{2}{*}{171} & \multirow{2}{*}{0,360} \\
\hline & $\mathrm{P}$ & 3,30 & 0,72 & & & \\
\hline \multirow{2}{*}{ Dispositivos multimedia interactivos } & $\mathrm{I}$ & 1,86 & 0,71 & \multirow{2}{*}{13,56} & \multirow{2}{*}{171} & \multirow{2}{*}{$0,000^{*}$} \\
\hline & $\mathrm{P}$ & 2,31 & 0,89 & & & \\
\hline \multicolumn{7}{|l|}{ Usos musicales } \\
\hline \multirow{2}{*}{ Reproductores de sonido } & $\mathrm{I}$ & 3,98 & 0,79 & \multirow{2}{*}{1,67} & \multirow{2}{*}{171} & \multirow{2}{*}{0,198} \\
\hline & $\mathrm{P}$ & 3,24 & 0,73 & & & \\
\hline \multirow{2}{*}{ Portales de vídeo musicales online } & $\mathrm{I}$ & 3,07 & 0,77 & \multirow{2}{*}{4,73} & \multirow{2}{*}{171} & \multirow{2}{*}{$0,031^{*}$} \\
\hline & $\mathrm{P}$ & 3,33 & 0,75 & & & \\
\hline \multirow{2}{*}{ Enciclopedias virtuales musicales } & $\mathrm{I}$ & 2,41 & 0,81 & 020 & 171 & 0649 \\
\hline & $\mathrm{P}$ & 2,47 & 0,96 & 0,20 & $1 / 1$ & 0,049 \\
\hline Disnositivos multimedia interastives & $\mathrm{I}$ & 1,65 & 0,67 & 1463 & 171 & $0000 *$ \\
\hline Dispositıvos multimeala interactivos & $\mathrm{P}$ & 2,11 & 0,89 & 14,03 & $1 / 1$ & $0,000^{\top}$ \\
\hline Actitudes musicales & & & & & & \\
\hline Importante conocer múcica decde niño & $\mathrm{I}$ & 3,51 & 0,57 & 615 & 171 & $0014 *$ \\
\hline mportante conocer musica desce mino & $\mathrm{P}$ & 3,27 & 0,67 & 0,15 & $1 / 1$ & 0,014 \\
\hline Medio nara fomentar rolacionec & $\mathrm{I}$ & 2,81 & 0,80 & 452 & 171 & \\
\hline Medio para tomentar relaciones & $\mathrm{P}$ & 2,56 & 0,90 & 4,52 & $1 / 1$ & $0,040^{\top}$ \\
\hline Fácil acceso oracias a lac TIC & $\mathrm{I}$ & 3,53 & 0,66 & 122 & 171 & 0270 \\
\hline racil acceso gracias a ias $11 \mathrm{c}$ & $\mathrm{P}$ & 3,40 & 0,80 & 1,22 & $1 / 1$ & $0,2 / 0$ \\
\hline & $\mathrm{I}$ & 3,05 & 0,71 & 476 & 171 & \\
\hline Debe conservarse el folclore & $\mathrm{P}$ & 2,80 & 0,78 & $4, / 6$ & 171 & $0,030^{*}$ \\
\hline Usos y actitudes según estilo aprendizaj & & & & & & \\
\hline Me ousta buscar música en la red & $\mathrm{I}$ & 2,80 & 0,86 & & 171 & 0206 \\
\hline Ive gusta Duscar musica en ra red & $\mathrm{P}$ & 2,97 & 0,89 & 1,01 & $1 / 1$ & 0,206 \\
\hline & $\mathrm{I}$ & 2,25 & 0,93 & 041 & 171 & 0522 \\
\hline Descubro musicas nuevas a amigos & $\mathrm{P}$ & 2,35 & 1,01 & 0,41 & $1 / 1$ & $0,0<3$ \\
\hline Me ousta oroanizar tertulias musicales & $\mathrm{I}$ & 1,63 & 0,85 & 026 & 171 & 0607 \\
\hline The gusta organizar tertuinas musicales & $\mathrm{P}$ & 1,56 & 0,79 & $0, \angle 0$ & $1 / 1$ & $0,00 /$ \\
\hline & $\mathrm{I}$ & 2,13 & 0,80 & 712 & 171 & $0009 *$ \\
\hline Pienso que ios debates musicales & $\mathrm{P}$ & 2,65 & 0,79 & 1,12 & $1 / 1$ & 0,005 \\
\hline
\end{tabular}




\begin{tabular}{|c|c|c|c|c|c|c|}
\hline & & $\mathbf{M}$ & DT & $\mathbf{F}$ & gl & p \\
\hline \multirow{2}{*}{ Prefiero investigaciones musicales en solitario } & $\mathrm{I}$ & 2,06 & 0,87 & \multirow{2}{*}{1,12} & \multirow{2}{*}{171} & \multirow{2}{*}{0,290} \\
\hline & $\mathrm{P}$ & 1,92 & 0,84 & & & \\
\hline \multirow{2}{*}{ Actúo con intuición en comentarios musicales } & I & 2,19 & 0,74 & \multirow{2}{*}{2,45} & \multirow{2}{*}{171} & \multirow{2}{*}{0,119} \\
\hline & $\mathrm{P}$ & 2,01 & 0,77 & & & \\
\hline \multirow{2}{*}{ Me interesa opiniones musicales de otros } & $\mathrm{I}$ & 1,72 & 0,77 & \multirow{2}{*}{3,84} & \multirow{2}{*}{171} & \multirow{2}{*}{0,065} \\
\hline & $\mathrm{P}$ & 1,94 & 0,78 & & & \\
\hline \multirow{2}{*}{ Obtengo conclusiones en trabajos musicales } & $\mathrm{I}$ & 1,92 & 0,72 & \multirow{2}{*}{5,09} & \multirow{2}{*}{171} & \multirow{2}{*}{$0,025^{1}$} \\
\hline & $\mathrm{P}$ & 2,39 & 0,83 & & & \\
\hline
\end{tabular}

Tabla 2: Comparación de medias (ANOVA) de los conocimientos, usos, actitudes musicales y estilo de aprendizaje del alumnado universitario en cuanto a la especialidad (Grado de Maestro en Infantil y

Primaria)

2) Determinar si los conocimientos, usos y actitudes musicales relacionados con las TIC y el estilo de aprendizaje difieren en función del género de los estudiantes de dicha Facultad.

Se localizaron diferencias estadísticamente significativas en dos ítems: "Es fácil acceder a la música gracias a las TIC" ( $p=.008)$ y "Pienso que los debates musicales unen" $(p=.005)$. En el caso del primer ítem significativo, éste se situó en el subapartado actitudes a favor del género femenino $(M=3.52 ; D T=.68)$; mientras que el segundo ítem significativo, se halló en el subapartado uso y actitudes según estilo de aprendizaje a favor del género masculino $(M=3.00 ; D T=.73)$. La Tabla 3 muestra la comparación de medias por género.

\begin{tabular}{|c|c|c|c|c|c|c|}
\hline & & $\mathbf{M}$ & DT & $\mathbf{F}$ & gl & $\mathbf{p}$ \\
\hline \multicolumn{7}{|l|}{ Conocimientos musicales } \\
\hline \multirow{2}{*}{ Blog, wiki, foro para comentar música } & $\mathrm{M}$ & 1,98 & 0,90 & \multirow{2}{*}{2,51} & \multirow{2}{*}{171} & \multirow{2}{*}{0,115} \\
\hline & $\mathrm{V}$ & 2,30 & 0,97 & & & \\
\hline \multirow{2}{*}{ Medios para grabar compartir música } & $\mathrm{M}$ & 2,48 & 0,77 & \multirow{2}{*}{2,83} & \multirow{2}{*}{171} & \multirow{2}{*}{0,094} \\
\hline & $\mathrm{V}$ & 2,78 & 0,90 & & & \\
\hline \multirow{2}{*}{ Portales de vídeo musicales online } & $\mathrm{M}$ & 3,22 & 0,71 & \multirow{2}{*}{2,67} & \multirow{2}{*}{171} & \multirow{2}{*}{0,104} \\
\hline & $\mathrm{V}$ & 3,48 & 0,59 & & & \\
\hline \multirow{2}{*}{ Dispositivos multimedia interactivos } & $\mathrm{M}$ & 2,08 & 0,81 & \multirow{2}{*}{0,24} & \multirow{2}{*}{171} & \multirow{2}{*}{0,642} \\
\hline & $\mathrm{V}$ & 2,17 & 1,02 & & & \\
\hline \multicolumn{7}{|l|}{ Usos musicales } \\
\hline \multirow{2}{*}{ Reproductores de sonido } & $\mathrm{M}$ & 3,15 & 0,77 & \multirow{2}{*}{0,42} & \multirow{2}{*}{171} & \multirow{2}{*}{0,513} \\
\hline & $\mathrm{V}$ & 3,26 & 0,75 & & & \\
\hline \multirow{2}{*}{ Portales de vídeo musicales online } & $\mathrm{M}$ & 3,18 & 0,75 & \multirow{2}{*}{0,92} & \multirow{2}{*}{171} & \multirow{2}{*}{0,337} \\
\hline & $\mathrm{V}$ & 3,35 & 0,88 & & & \\
\hline \multirow{2}{*}{ Enciclopedias virtuales musicales } & $\mathrm{M}$ & 2,42 & 0,88 & \multirow{2}{*}{0,50} & \multirow{2}{*}{171} & 0478 \\
\hline & $\mathrm{V}$ & 2,57 & 0,94 & & & $0,4 / 8$ \\
\hline Dicnocitivoc multimedia interastivos & $\mathrm{M}$ & 1,87 & 0,79 & 0.91 & 171 & 0340 \\
\hline Dispositıvos multimedia interactivos & $\mathrm{V}$ & 2,05 & 0,99 & 0,91 & $1 / 1$ & 0,340 \\
\hline Actitudes musicales & & & & & & \\
\hline Imnortante conocer múcica desde niño & $\mathrm{M}$ & 3,41 & 0,62 & 183 & 171 & 0177 \\
\hline importante conocer musica desce nino & $\mathrm{V}$ & 3,22 & 0,67 & 1,83 & $1 / 1$ & $0,1 / 7$ \\
\hline Modio nara fomentar rolacionec & $\mathrm{M}$ & 2,66 & 0,85 & 037 & 171 & 0513 \\
\hline medo para iomentar reraciones & $\mathrm{V}$ & 2,78 & 0,90 & $0,3 /$ & $1 / 1$ & 0,543 \\
\hline Fácil acceso đracias a lac TIC & $\mathrm{M}$ & 3,52 & 0,68 & 709 & 171 & \\
\hline Facıl acceso gracias a las II & $\mathrm{V}$ & 3,09 & 0,99 & 1,09 & $1 / 1$ & $0,008^{\top}$ \\
\hline & $\mathrm{M}$ & 2,95 & 0,74 & 2.30 & 171 & 0.131 \\
\hline Debe conservarse el folclore & $\mathrm{V}$ & 2,70 & ,82 & 2,30 & $1 / 1$ & 0,131 \\
\hline Usos y actitudes según estilo aprendiza & & & & & & \\
\hline & $\mathrm{M}$ & 2,90 & 0,89 & & & \\
\hline Me gusta buscar musica en la red & $\mathrm{V}$ & 2,78 & 0,85 & 0,34 & $1 / 1$ & 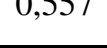 \\
\hline Descubro músicas nuevas a amions & $\mathrm{M}$ & 2,32 & 0,98 & & 171 & 0655 \\
\hline Descubro musicas nuevas a amıgos & $\mathrm{V}$ & 2,22 & 0,90 & 0,20 & $1 / 1$ & 0,655 \\
\hline
\end{tabular}

${ }^{1}$ Significativo al 5\% o superior. 


\begin{tabular}{|c|c|c|c|c|c|c|}
\hline & & $\mathbf{M}$ & DT & $\mathbf{F}$ & gl & $\mathbf{p}$ \\
\hline \multirow{2}{*}{ Me gusta organizar tertulias musicales } & $\mathrm{M}$ & 1,56 & 0,80 & \multirow{2}{*}{1,41} & \multirow{2}{*}{171} & \multirow{2}{*}{0,236} \\
\hline & $\mathrm{V}$ & 1,78 & 0,95 & & & \\
\hline \multirow{2}{*}{ Pienso que los debates musicales unen } & $\mathrm{M}$ & 2,53 & 0,79 & \multirow{2}{*}{7,51} & \multirow{2}{*}{171} & \multirow{2}{*}{0,005} \\
\hline & $\mathrm{V}$ & 3,00 & 0,73 & & & \\
\hline \multirow{2}{*}{ Prefiero investigaciones musicales en solitario } & $\mathrm{M}$ & 1,99 & 0,83 & \multirow{2}{*}{0,00} & \multirow{2}{*}{171} & \multirow{2}{*}{0,945} \\
\hline & $\mathrm{V}$ & 2,00 & 1,00 & & & \\
\hline \multirow{2}{*}{ Actúo con intuición en comentarios musicales } & $\mathrm{M}$ & 2,07 & 0,76 & \multirow{2}{*}{1,20} & \multirow{2}{*}{171} & \multirow{2}{*}{0,275} \\
\hline & $\mathrm{V}$ & 2,26 & 0,75 & & & \\
\hline \multirow{2}{*}{ Me interesa opiniones musicales de otros } & $\mathrm{M}$ & 1,79 & 0,75 & \multirow{2}{*}{3,76} & \multirow{2}{*}{171} & \multirow{2}{*}{0,054} \\
\hline & $\mathrm{V}$ & 2,13 & 0,92 & & & \\
\hline \multirow{2}{*}{ Obtengo conclusiones en trabajos musicales } & $\mathrm{M}$ & 2,45 & 0,79 & \multirow{2}{*}{0,02} & \multirow{2}{*}{171} & \multirow{2}{*}{0,871} \\
\hline & $\mathrm{V}$ & 2,48 & 0,73 & & & \\
\hline
\end{tabular}

Tabla 3: Comparación de medias (ANOVA) de los conocimientos, usos, actitudes musicales y estilo de aprendizaje del alumnado universitario en cuanto al género

\section{DISCUSIÓN Y CONCLUSIONES}

El análisis de los resultados permitió comprobar la existencia de diferencias estadísticamente significativas en los conocimientos, usos, actitudes musicales y estilos de aprendizaje analizados en cuanto a la especialidad del Grado de Maestro, pero no respecto al género. Por especialidad, los resultados desprendieron una división en la respuesta de los participantes. Por un lado, los estudiantes del Grado de Educación Primaria manifestaron unos conocimientos y usos musicales significativamente mayores que los del Grado de Infantil, en relación con el conocimiento y uso que hacen de dispositivos multimedia interactivos y de portales de vídeo musicales online. Este hecho revela un interés hacia las TIC superior en los estudiantes de Primaria probablemente debido a que, como señalan Bauer, McAllister y Reese, 2003, conceden un papel clave a estas herramientas en su formación y en su aplicación práctica en el aula de música. Por otro lado, el alumnado de Educación Infantil puntuó por encima en actitudes musicales reconociendo que es importante conocer la música desde niño, que la música es un medio para fomentar relaciones entre los compañeros de la clase y que debe conservarse en sus aspectos tradicionales o folclóricos. Este resultado puede explicarse por una mayor sensibilidad presente en los estudiantes de Educación Infantil, ya que su ámbito de actuación abarca a niños de edades tempranas, por lo que es fundamental para ellos iniciar la música lo antes posible y emplearla como medio de relación interpersonal y afectiva en las clases de Educación Infantil (Tapscott, 2009).

En cuanto a usos musicales y actitudes ante la música según el estilo de aprendizaje, los resultados significativos evidenciaron que los estudiantes de Primaria tenían un estilo de aprendizaje reflexivoteórico de acuerdo a su respuesta a los ítems identificados con estos estilos como fueron "Pienso que los debates musicales unen" y “Obtengo conclusiones en trabajos musicales”. Este resultado se encuentra en la línea con otras investigaciones (Alonso \& Gallego, 2003), que identifican el estilo reflexivo-teórico como el predominante en el alumnado universitario. No obstante, la falta de un mayor número de puntuaciones estadísticamente significativas en el presente estudio no permite concluir que el estilo de aprendizaje esté vinculado con los usos y actitudes musicales del alumnado participante, si bien estos resultados contribuyen a completar el marco empírico y pueden servir de punto de partida para otras investigaciones futuras sobre consumo musical en estudiantes universitarios, en línea con los estudios de Aranguren (2011).

Atendiendo a los resultados obtenidos, cabe reflexionar cómo el desarrollo integral de las personas y, más concretamente de nuestros estudiantes, pasa por la necesaria movilización de conocimientos, destrezas y actitudes que conforman la competencia musical, tal y como señalan Ricoy, Feliz y

\footnotetext{
${ }^{2}$ Significativo al 5\% o superior.
} 
Sevillano (2010) o De Moya y Cózar (2013). En la sociedad actual, caracterizada por la tecnificación y la presencia de las TIC en todos los ámbitos de la vida y de manera particular en educación, requiere emprender procesos de autorreflexión en nuestros estudiantes para afrontar con éxito los retos de una sociedad cada vez más virtualizada. En el caso de la competencia musical, ésta se ha visto influenciada de manera significativa por los nuevos usos de consumo derivados de la expansión de las TIC (Barry, 2004; Enders, 2000). Las nuevas tecnologías, sea cuales sean sus múltiples formatos y versiones (cada vez más novedosos y cambiantes por el negocio y la demanda de los mercados) amplían sus utilidades con la ilusoria idea de que son herramientas imprescindibles en nuestras vidas. En cualquier caso, hay que reconocer que, ya sea por intereses mercantiles, empresariales, sociológicos o educativos, entre otros, las TIC han conquistado incuestionablemente un amplio espacio, que va en aumento, en la vida común y diaria de gran parte de la población, y que repercute especialmente en los hábitos de consumo musical de los más jóvenes.

\section{LIMITACIONES Y PROSPECTIVA}

Los resultados recogidos en este estudio obedecen a un contexto determinado y a unas características particulares de los estudiantes, hecho que limita la extrapolación de los hallazgos. Del mismo modo, el procedimiento de selección de la muestra, efectuado por accesibilidad muestral, restringe la generalización de los resultados. Igualmente, la descompensación de la muestra respecto al género, una circunstancia que no podía solventarse al utilizar la totalidad de los participantes disponibles y que era superior en género femenino, puede constituir un elemento de sesgo para la investigación. Aun así, se planteó como objetivo de estudio teniendo en cuenta esta limitación en aras de una mayor profundización del fenómeno abordado. En esta línea, hay que considerar el perfil del alumnado analizado, que se ajusta a los objetivos planteados, es decir, corresponde con una población cronológicamente joven con un nivel de estudios alto que consume habitualmente música, y por lo tanto, es de notable interés para el presente estudio. No obstante, como limitación debe señalarse la ausencia de una mayor profundización entre la vinculación de estilos de aprendizaje y usos y actitudes musicales a través de las TIC. A pesar de que las preguntas del cuestionario USMUS, uno de los dos suministrados a los estudiantes participantes en la investigación, intentan abordar esta cuestión, es necesario emprender nuevos trabajos de investigación que ofrezcan explicaciones más profundas en cuanto al fenómeno estudiado.

A pesar de las numerosas implicaciones que tiene este tema y de su interés para el ámbito científico, todavía existe una escasez de investigaciones, que no avanzan tan rápidamente como las nuevas tecnologías, capaces de convertirse en un fenómeno vital y casi imprescindible, con un impacto sociológico tan destacado, que no es posible calibrarlo en toda su magnitud e intensidad (Anderson \& Tracey, 2002). Por este motivo, pretendemos que el presente trabajo sirva de ayuda para reflexionar hacia dónde pueden encaminarse, en un futuro inmediato, los usos, costumbres y actitudes, la competencia musical, de los estudiantes de Grado de Maestro. A fin de cuentas, lo tecnológico vive en un permanente proceso de cambio, si bien la inclinación al consumo musical, por parte de las personas, siempre ha sido una constante histórica.

\section{Referencias}

Alonso, C. M. \& Gallego, D. J. (2003). Cómo diagnosticar y mejorar los estilos de aprendizaje. Madrid: UNED.

Anderson, B. \& Tracey, K. (2002). Digital living: The impact of the Internet on everyday life. En B. Wellman \& C. Haythornwaite (Eds.), The internet in everyday life. Londres: Blackwell.

Aranguren, A. (2011). El consumo musical adolescente como fuente de estrategias didácticas. Eufonía, 53, 25-33. 
Barry, N. (2004). University music education student perceptions and attitudes about instructional technology. Journal of Technology in Music Learning, 2(2), 2-20.

Bauer, W., McAllister, P., \& Reese, S. (2003). Transforming music teaching via technology: The role of professional development. Journal of Research in Music Education, 51(4), 289-301. doi: $10.2307 / 3345656$

Bangemann, M. (Ed.) (1994). Europa y la Sociedad Global de la Información. Recomendaciones al Consejo Europeo. Bruselas: European Council.

Beckstead, D. (2001). Will technology transform music education? Music Educators Journal, 87(6), 44-49. doi: $10.2307 / 3399692$

Bell, D. (1973). The coming of post-industrial society. A venture in social forecasting. Nueva York: Basic Books.

Bermell, M. A. \& Alonso, V. (2012). La educación musical en la sociedad del futuro. Música y Educación, 25(92), 50-57.

Blardony, S. (2006). La formación permanente del profesorado de música a través de las nuevas tecnologías. Música y Educación, 19(67), 151-157.

Buckingham, D. (2002). The electronic generation? Children and new media. En L. Lievrouw \& S. Livingstone (Eds.), The handbook of new media. Londres: Sage.

Burnard, P. (2007). Reframing creativity and technology: promoting pedagogic change in music education. Journal of Music, Technology and Education, 1(1), 37-55. doi: 10.1386/jmte.1.1.37/1

Campos, J. L. (2008). Cuando la música cruzó la frontera digital. Aproximación al cambio tecnológico y cultural de la comunicación musical. Madrid: Biblioteca Nueva.

Cardona, M. C. (2002). Introducción a los métodos de investigación en educación. Madrid: EOS.

Castells, M. (1998). La era de la información. Economía sociedad y cultura. Fin del milenio. Madrid: Alianza.

Cózar, R. \& De Moya, M. V. (2013). Las TIC en el aula desde un enfoque multidisciplinar. Aplicaciones prácticas. Barcelona: Octaedro.

Cuban, L. (2001). Oversold and underused: Computers in the classroom. Cambridge-Massachusetts: Harvard University Press.

De Aguilera, M. \& Sedeño, A. (2008). Comunicación y música II. Tecnología y audiencias. Barcelona: UOC.

De Moya, M. V., Hernández, J. A., Hernández, J. R., \& Cózar, R. (2011). Análisis de los estilos de aprendizaje y las TIC en la formación personal del alumnado universitario a través del cuestionario REATIC. Revista de Investigación Educativa, 29(1), 137-156.

Eco, U. (2001). La definición del arte. Madrid: Destino.

Enders, B. (2000). Musical education and the new media: The current situation and perspectives for the future. En H. Braun (Ed.), Music and technology in the twentieth century (pp. 223-238). Baltimore: The Johns Hopkins University Press.

Gértrudix, F. \& Gértrudix, M. (2014). Herramientas y recursos para la creación y consumo musical en la web 2.0. Aplicaciones y potencialidades educativas. Educación XX1, 17(2), 313-336. doi: 10.5944/educxx1.17.2.11493

Giráldez, A. (2005). Internet y educación musical. Barcelona: Graó.

Herrera, L. Cremades, R., \& Lorenzo, O. (2010). Preferencias musicales de los estudiantes de 
Educación Secundaria Obligatoria: influencia de la educación formal e informal. Cultura y Educación, 22(1), 37-51.

Hernández, J. A. (2013). Creación artística con software libre. En R. Cózar y M. V. De Moya (Eds.), Las TIC en el aula desde un enfoque multidisciplinar. Aplicaciones prácticas (pp. 139-156). Barcelona: Octaedro.

Hernández, J. R. (2013). Las TIC en el aula 2.0. Una visión práctica acerca de su integración. En R. Cózar \& M. V. De Moya (Eds.), Las TIC en el aula desde un enfoque multidisciplinar. Aplicaciones prácticas (pp. 29-44). Barcelona: Octaedro.

Hernández, J. A., Hernández, J. R., De Moya, M. V. \& Cózar, R. (2014). La educación musical competencial en España: ¿Necesidad o deseo? Revista Electrónica Educare, 18(3), 237-249. doi: $10.15359 /$ ree.18-3.14

Ho, W. (2004). Attitudes towards information technology in music learning among Hong Kong Chinese boys and girls. British Journal of Music Education, 21(2), 143-161. doi: 10.1017/S0265051704005662

Kerr, S. T. (2004). Toward a Sociology of educational technology. En D. Jonassen (Ed.), Handbook of research on educational communications and technology. Nahwah, New Jersey: Erlbaum.

Livingstone, S. (2009). Children and the Internet: Great expectations, challenging realities. Cambridge: Policy Press.

Majó, J. \& Marqués, P. (2002). La revolución educativa en la era de Internet. Barcelona: CissPraxis.

Machlup, F. (1962). The Production and Distribution of Knowledge in the United States. Princeton: Princeton University Press.

McMillan, K., Honey, M. \& Mandinach, E. (2005). A retrospective on twenty years of education technology policy. Journal educational computing research, 32(3), 279-307.

Mir, B. (2010). Competencias digitales: Conocimientos, habilidades y actitudes para la sociedad red. Servei de Tecnologies per a l’Aprenentatge i el Coneixement, Barcelona: UIMPB.

Ramos, P. (2011). Texto digital e internet: Hacia una nueva legitimación y uso de la bibliografía científica en educación musical. Revista Electrónica Complutense de Investigación en Educación Musical, 8(2), 1-13. doi: 10.5209/rev_RECI.2011.n8.38029

Ricoy, M. C., Feliz, T. \& Sevillano, M. L. (2010). Competencias para la utilización de las herramientas digitales en la sociedad de la información. Educación XX1, 13(1), 199-219.

Rusinek, G. (2004). Aprendizaje musical significativo. Revista Electrónica Complutense de Investigación en Educación Musical, 1(5). Disponible en http://revistas.ucm.es/index.php/ RECI/article/ view/9622

Ruthmann, S. A., Tobias, E. S., Randles, C. \& Thibeault, M. (2015). Is it the technology? Challenging technological determinism in music education. En C. Randles (Ed.), Music education: Navegating the future (pp.122-138). Nueva York: Routledge.

Savage, J. (2005). Working towards a theory for music technologies in the classroom: How pupils engage with and organize sounds with new technologies. British Journal of Music Education, 22(2), 167-180. doi: 10.1017/S0265051705006133

Solomando, A., Almerich, G. \& Fossati, R. (2012). Uso de las tecnologías de la información y la comunicación en los estudiantes de Pedagogía Musical. En Actas del II Congreso de Educación e Investigación Musical (pp. 94-101). Madrid: Sociedad para la Educación Musical del Estado Español, Instituto de Educación Musical y Universidad Rey Juan Carlos de Madrid. 
Storr, A. (2008). La música y la mente. Barcelona: Paidós.

Suárez, J. M., Almerich, G., Gargallo, B. \& Aliaga, F. M. (2013). Las competencias del profesorado en TIC: Estructura básica. Educación XX1, 16(1), 39-62.

Tapscott, D. (2009). Grown up digital: How the net generation is changing your world. Nueva York: McGraw-Hill.

Torres, L. (2010). Las TIC en el aula de educación musical. Bases metodológicas y posibilidades prácticas. Sevilla: MAD.

Touraine, A. (1969). Sociología de la acción. Barcelona: Ariel. 